\title{
Budget impact analysis of sFlt-1/PIGF ratio as prediction test in Italian women with suspected preeclampsia
}

Tiziana Frusca 1, Maria-Teresa Gervasi 2, Davide Paolini 3, Matteo Dionisi 3, Francesca Ferre 4 5, Irene Cetin 6

\section{Affiliations}

- 1 Department of Obstetrics and Gynecology, University of Parma, Parma, Italy.

- 2 Azienda Ospedaliera di Padova, Padova, Italy.

- 3 Roche Diagnostics, Monza, Italy.

- 4 Scuola Superiore Sant'Anna, Istituto di Management - Laboratorio Management e Sanità , Pisa , Italy.

- 5 Temas srl, Milan, Italy, and.

- 6 Department of Biomedical and Clinical Sciences "Luigi Sacco", University of Milan , Milan , Italy.

\begin{abstract}
Introduction: Preeclampsia (PE) is a pregnancy disease which represents a leading cause of maternal and perinatal mortality and morbidity. Accurate prediction of PE risk could provide an increase in health benefits and better patient management.

Objective: To estimate the economic impact of introducing Elecsys sFlt-1/PIGF ratio test, in addition to standard practice, for the prediction of PE in women with suspected PE in the Italian National Health Service (INHS).

Methods: A decision tree model has been developed to simulate the progression of a cohort of pregnant women from the first presentation of clinical suspicion of PE in the second and third trimesters until delivery. The model provides an estimation of the financial impact of introducing sFlt-1/PIGF versus standard practice. Clinical inputs have been derived from PROGNOSIS study and from literature review, and validated by National Clinical Experts. Resources and unit costs have been obtained from Italian-specific sources.
\end{abstract}

Results: Healthcare costs associated with the management of a pregnant woman with clinical suspicion of PE equal $€ 2384$ when following standard practice versus $€ 1714$ using sFlt-1/PlGF ratio test.

Conclusions: Introduction of sFlt-1/PlGF into hospital practice is cost-saving. Savings are generated primarily through improvement in diagnostic accuracy and reduction in unnecessary hospitalization for women before PE's onset. 


\section{Keywords:}

Preeclampsia

sFlt-1

PIGF

budget impact

sFlt

\section{Introduction}

Preeclampsia (PE) is a hypertensive disorder of pregnancy (HDP) traditionally diagnosed by the combined presence of increased blood pressure (systolic blood pressure $\geq 140 \mathrm{~mm} \mathrm{Hg}$ and/or diastolic blood pressure $\geq 90 \mathrm{~mm} \mathrm{Hg}$ ) and proteinuria ( $\geq 0.3 \mathrm{~g} / 24$ hours). PE affects $2-8 \%$ of pregnancies and along with the other hypertensive disorders is a major cause of maternal and perinatal mortality and morbidity worldwide $[\underline{1}, \underline{2}]$.

Assessment of initial presentation of PE is difficult because it is often asymptomatic until late stage of the disease and involves multi-organ dysfunctions [3]. Early diagnosis requires multiple reading of clinical and laboratory tests and monitoring of patients by expert gynecologists, obstetricians and/or midwives. International and National Guidelines suggest to perform specific investigations for the diagnosis of PE such as the evaluation of blood pressure and proteinuria that are considered the standard diagnostic test, along with other maternal tests as full blood count, liver function, renal function and coagulation test. Also uterine artery Doppler and ultrasonography assessment of fetal growth and placental function are suggested [3-5].

According to PE severity, patients may be recommended routine visits in outpatient setting or require hospitalization in order to receive appropriate clinical diagnosis and continuous monitoring. Recently, evaluation of placental growth factor (PlGF) and quantification of the ratio between sFlt-1 and PlGF in the second and third trimesters of pregnancy has been shown to predict the subsequent development of PE [6-9], allowing a more accurate prediction of the onset of disorder and better patient management.

In Europe, the automated measurement of the sFlt-1/PlGF ratio has been approved for aid in the diagnosis of PE [10]. Recently, PROGNOSIS - a global multicenter non-interventional prospective

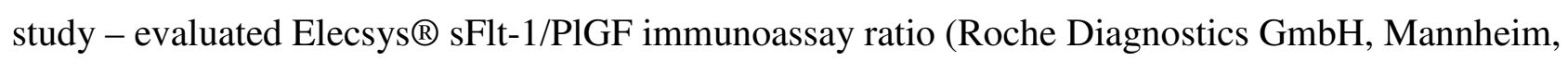
Germany) as the first fully automated immunoassay for the detection of PE biomarkers in maternal serum after 20 weeks of gestation. The test was able to show a negative predictive value of $99.3 \%$ for ruling out PE within 1 week [11]. Clinical data therefore suggest that this test in conjunction with other clinical findings aids the prediction of $\mathrm{PE}$ and prevent over-diagnosis and over-treatment of women with suspected PE [12].

Improved diagnostic tests have the potential to increase accuracy levels, better manage patients according to appropriate level of risk and reduce unnecessary healthcare costs. When new health 
technologies become available, decision-makers usually assess benefits and costs of new technologies in order to appreciate the increased value for the patients and perform estimates of the financial sustainability for the healthcare system. Indeed, the use of economic evaluation for the assessment of new health technologies is becoming increasingly important to support healthcare decisions when resources are scarce and health needs are infinite. The purpose of this analysis was to estimate the financial impact of introducing the sFlt-1/PIGF test into hospital practice for the prediction of PE in women with the suspicion of this disease in addition to standard practice, in the Italian National Health Service (INHS).

\section{Methods}

An economic model was developed from the INHS payer's perspective to estimate the net financial impact of the innovative sFlt-1/PIGF test in comparison to the current clinical practice. Net impact was calculated on the difference between direct healthcare costs (hospital, outpatient and drugs) associated with the diagnosis and management of women with sign and symptoms of suspected $\mathrm{PE}^{1}$, when using the sFlt-1/PIGF (test scenario) and current clinical practice (no test scenario). A 5year-long budget impact estimation has been performed including a 3\% discount rate [13]. In order to check the robustness of the results, a sensitivity analysis with a variation of $\pm 20 \%$ on input assumptions has been conducted in comparison to the baseline.

Model structure

The decision tree model simulates the progression of a pregnant woman with HDP through a treatment pathway that is determined by the assessment of the risk of developing PE and the consequent decision to hospitalize [High] or manage the pregnancy in an outpatient setting [Intermediate/Low] (Figure 1). The model includes direct healthcare resources used from the first presentation of clinical suspicion of $\mathrm{PE}^{1}$ (gestational week $24+0$ until $36+6$ ) to delivery (full-term 40 weeks). 
Figure 1. Decision tree model structure.

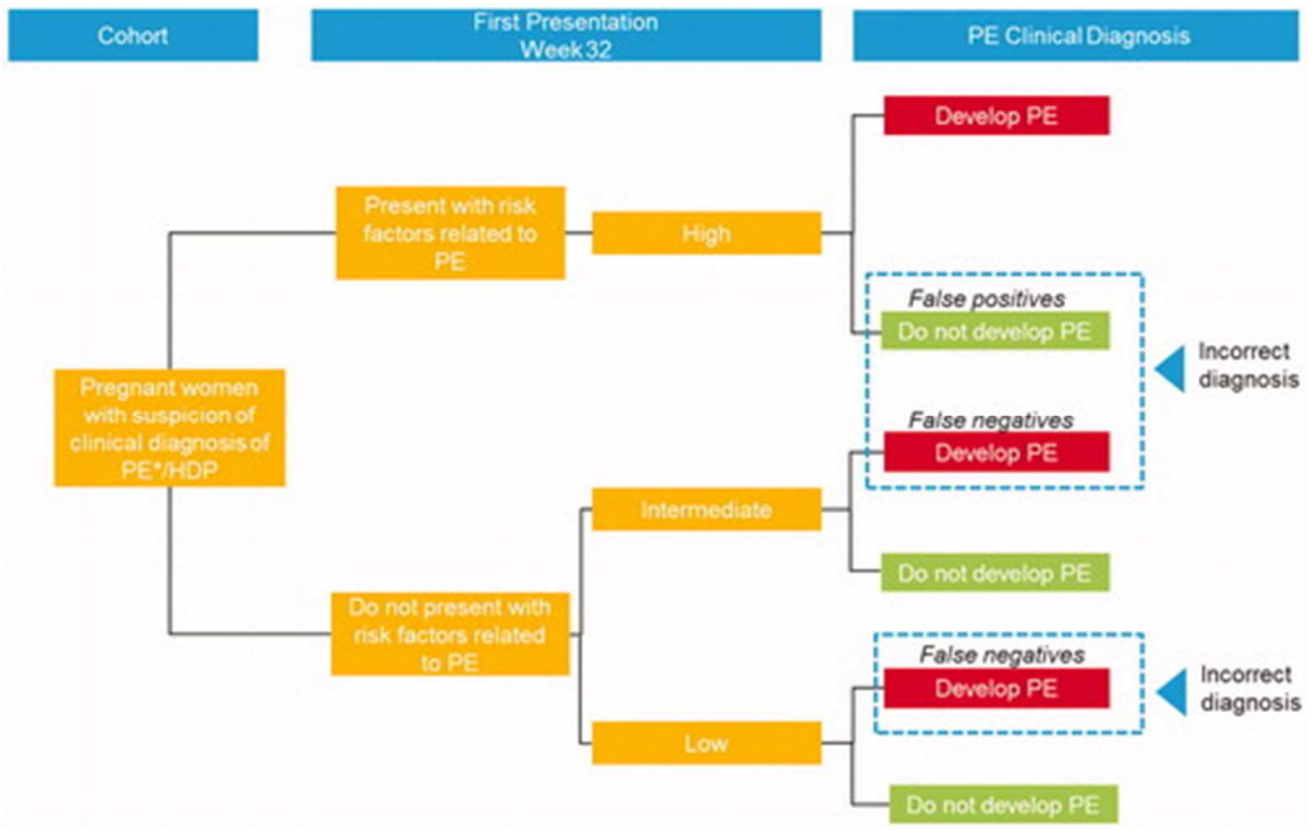

At the clinical visit, pregnant women are classified as low, intermediate or high risk of PE's onset. Intermediate/low-risk gestational hypertensions are monitored in outpatient settings biweekly or monthly until PE was clinically diagnosed. High-risk PE patients are recommended to undergo a full clinical assessment in hospital setting and weekly outpatient visit until PE could be clinically diagnosed. The model assumes the introduction of the sFlt-1/PlGF test during the first presentation with the objective to provide a better diagnosis and targeted management of pregnant women with suspected $\mathrm{PE}^{1}$. In cases of confirmed diagnosis of $\mathrm{PE}$, women were hospitalized until delivery. Data and modeling assumption

Target population included in the model considers pregnant women with suspected $\mathrm{PE}^{1}$. The cohort of patients included in the model was equal to 49455 pregnant women and was calculated as number of alive births per year in Italy [14] considering year 2014 (494 550) multiplied by HDP prevalence rate that ranges from $5 \%$ to $15 \%$ with a median of $10 \%$ [15-17], following cohorts' estimation considered an annual growth rate in female population of $0.68 \%{ }^{2}$ [14].

The economic model used patient-level data derived from the PROGNOSIS study [11] to identify progression of PE's risk, onset, complications, length of stay (LOS) in hospital and the sFlt-1/PIGF test at baseline. In the current scenario, which does not include the sFlt-1/PIGF test, interviews to three National Clinical Experts (MDs Gynecology and Obstetrics) were carried out to validate assumptions and to understand patient management practices.

According to National Guidelines and National Experts' opinion, the baseline examination to rule out patients with suspected PE (occurring between 28th and 32nd gestational week) included measurement of blood pressure, urine analysis, ultrasound evaluation of fetal growth and Doppler 
sonography, evaluation of full blood count, creatinine, lactate dehydrogenase (LDH) test and aspartate aminotransferase (AST). In the economic model, at first presentation, patients who are not admitted into hospital are assumed to be managed in an outpatient setting. Outpatient's resource use was modeled by distinguishing low and intermediate intensity management based on risk of PE onset (Table 1). 
Table 1. Patient management options for women with suspected PE.

\begin{tabular}{|c|c|c|}
\hline \multicolumn{2}{|c|}{ Non-hospitalized } & \multirow{2}{*}{$\begin{array}{c}\text { Hospitalized } \\
\text { High }\end{array}$} \\
\hline Low & Intermediate & \\
\hline $\begin{array}{l}\text { Women are managed in outpatient setting } \\
\text { with an average once every } 2 \text { weeks } \\
\text { appointment/evaluation and can also be } \\
\text { managed by domiciliary care. }\end{array}$ & $\begin{array}{l}\text { Women are managed in outpatient } \\
\text { setting with an average weekly } \\
\text { appointment/evaluation. }\end{array}$ & $\begin{array}{l}\text { Women are managed in } \\
\text { inpatient setting (always } \\
\text { ordinary hospital regimen). }\end{array}$ \\
\hline $\begin{array}{l}\text { They are usually women with pre- } \\
\text { pregnancy hypertension, high body mass } \\
\text { index, autoimmune diseases. }\end{array}$ & $\begin{array}{l}\text { They are usually women with } \\
\text { hypertension around } 12 \text { weeks of } \\
\text { pregnancy. }\end{array}$ & $\begin{array}{l}\text { They are usually women with } \\
\text { hypertension after } 20 \text { weeks of } \\
\text { pregnancy. }\end{array}$ \\
\hline Routine tests include: & Routine tests include: & Routine tests include: \\
\hline - Gynecologic visit & - Gynecologic visit & - Gynecologic visit \\
\hline - Blood test (blood count) & - Blood test (blood count) & - Blood test (blood count) \\
\hline - Blood pressure & - Blood pressure & - Blood pressure \\
\hline - Urine test & - Urine test & - Urine test \\
\hline - Creatinine test (for kidney function) & $\begin{array}{l}\text { - Creatinine test (for kidney } \\
\text { function) }\end{array}$ & $\begin{array}{l}\text { - Creatinine test (for } \\
\text { kidney function) }\end{array}$ \\
\hline - Bilirubin & - Bilirubin & - Bilirubin \\
\hline - Transaminases & - Transaminases & - Transaminases \\
\hline - Antithrombin & - Antithrombin & - Antithrombin \\
\hline - Fetal echocardiography & - Fetal echocardiography & - Fetal echocardiography \\
\hline - Electrolytes test $(\mathrm{Ca}, \mathrm{Cl}, \mathrm{P}, \mathrm{Na})$ & - Electrolytes test $(\mathrm{Ca}, \mathrm{Cl}, \mathrm{P}, \mathrm{Na})$ & $\begin{array}{c}\text { - Electrolytes test }(\mathrm{Ca}, \mathrm{Cl} \text {, } \\
\mathrm{P}, \mathrm{Na})\end{array}$ \\
\hline $\begin{array}{l}\text { At the first appointment, the color } \\
\text { Doppler sonography is a common test }\end{array}$ & $\begin{array}{l}\text { At the first appointment, the color } \\
\text { Doppler sonography is a common test }\end{array}$ & $\begin{array}{l}\text { At the first appointment the } \\
\text { color Doppler sonography is a } \\
\text { common test }\end{array}$ \\
\hline $\begin{array}{l}\text { Women can receive pharmacological } \\
\text { treatment (nifedipine is the first choice } 1 / 2 \\
\text { times per day, followed by labetalol) }\end{array}$ & $\begin{array}{l}\text { Women can receive pharmacological } \\
\text { treatment (nifedipine is the first choice } \\
1 / 2 \text { times per day, followed by labetalol) }\end{array}$ & $\begin{array}{l}\text { Women can also receive } \\
\text { pharmacological treatment for } \\
\text { hypertension (nifedipine is the } \\
\text { first choice } 1 / 2 \text { times per day, } \\
\text { followed by labetalol) and } \\
\text { betamethasone }(12 \mathrm{mg}) \text { and } \\
\text { magnesium sulfate }(4 \mathrm{~g} \\
\text { intravenous and } 1 \mathrm{~g} \\
\text { maintenance) in order to avoid } \\
\text { fetal complications }\end{array}$ \\
\hline
\end{tabular}


In the test scenario, women are classified into three categories based on the sFlt-1/P1GF ratio test results. A negative test result (ratio $\leq 38$ ) is able to rule-out PE within 1 week; a positive test result (ratio >38-85) predicts the rule-in of PE within 4 weeks; and a positive test result (ratio >85) indicates that the likelihood of PE is high and hospitalization together with the use of corticosteroids are highly recommended. The risk of PE and probability of hospitalization is expected to be positively correlated with increasing values of the sFlt-1/PlGF ratio. Analysis of the test's information from the PROGNOSIS study showed that for the women with a ratio $>85 \%$ $(13.2 \%), 64.8 \%$ of them were hospitalized. For the women with a ratio value in the range of $38-85$ $(10.7 \%), 55.4 \%$ were hospitalized. For the women with a ratio $\leq 38(76.1 \%)$, in accordance to National Experts' Opinion, it has been assumed that the 5\% would be hospitalized. Moreover, the model assumes that re-testing has to be performed 2 weeks after the initial test for women with negative result of the first test, have not presented with PE in the 2 weeks following their initial appointment and have not been hospitalized but do present at least one clinical sign of $\mathrm{PE}^{\underline{3}}(81.4 \%$ of women were eligible for re-test) [18].

The model also includes the cost of treatment considering complications such as an emergency admission and neonatal intensive care unit (nICU) admission for preterm births. Both costs have been calculated accordingly to the treatment management level, admission rates and LOS. In absence of evidence of the effect of test adoption on these costs, the model assumes the same economic impact for both test and no test scenario.

Costs

The economic evaluation considers all direct healthcare costs associated with test acquisition, diagnosis of pre-PE, hospitalization and outpatient management for women included in the model. The resources used for each management level are based on national and international guidelines for the management of women with hypertension in pregnancy $[\underline{3-5,15]}$ and validated through National Expert Opinions interviews.

The cost for the administration of the automated sFlt-1/PIGF test ( $€ 50.12$ ) was obtained from the only available regional tariffs established by the region of Liguria [19]. Hospitalization costs were estimated following the national diagnosis-related group (DRG)-based reimbursement system (version 24 of DRG-Grouper); admission codes used were DRG 384 corresponding to "Other prenatal diagnosis without medical complications" and DRG 383 corresponding to "Other prenatal diagnosis with medical complications". The model considers a weighted average cost of $€ 862.80$ [20] per hospitalized patient, where the majority of hospital admissions do not have medical complications. Consumption of healthcare resources related to first presentation (laboratory and diagnostic tests) and to outpatient management (low and intermediate levels) is coded using the 
national tariffs established by the Ministerial Decree of 18 October 2012 [20]. The unitary cost (exfactory price) of the drug for antihypertensive medication (nifedipine) is obtained from the Telematic Pharmaceutical Compendium 2015. The model also includes costs for complications which refer to emergency admissions and nICU since the risk of very low/low birth weight and preterm births increase with PE status [21]. The model uses the costs sustained by international countries as proxy for the economic impact associated with complications, that is, because national reimbursement tariffs for those services do not exist. Emergency admission ( $€ 1471)$ is obtained

from Payment by Results in the NHS (2014) $)^{4}$ [22], whereas nICU's cost per day (€1383) is acquired from a recent review of nICU's costs in four European countries including Italy [23].

\section{Results}

Overall cost difference year 1

The introduction of the sFlt-1/PIGF test in addition to standard practice reduces overall healthcare costs by $28 \%$ in the first year versus standard practice alone (from $€ 117.924 .952$ to $€ 84.764 .875$ ). The inclusion of the test as an additional diagnostic tool for PE risk detection has a major impact in reducing healthcare costs related to management of patients before PE onset. In particular, the test is able to reduce $69.5 \%$ of unnecessary patient's hospitalizations before PE onset allowing a better stratification of the risk, with a shift in management of patients from high to intermediate and low intensity level when patients do not develop PE (Table 2). 
Table 2. Overall cost difference 1 year (test-no test scenarios).

\begin{tabular}{|c|c|c|c|c|c|}
\hline & \multicolumn{2}{|c|}{ Costs } & \multirow[b]{2}{*}{$\Delta$ Costs } & \multicolumn{2}{|c|}{ Patients } \\
\hline & No test scenario & Test scenario & & No test scenario & $\begin{array}{c}\text { Test } \\
\text { scenario }\end{array}$ \\
\hline $\begin{array}{c}\text { Initial } \\
\text { appointment }\end{array}$ & $€ 4.464 .797$ & $€ 4.464 .797$ & $0 \%$ & 49455 & 49455 \\
\hline Test & - & $€ 2.478 .685$ & - & 0 & 49455 \\
\hline Retest & - & $€ 1.405 .603$ & - & 0 & 28045 \\
\hline $\begin{array}{c}\text { Pre-PE } \\
\text { management }\end{array}$ & $€ 39.203 .272$ & $€ 15.537 .517$ & $-60 \%$ & 49455 & 49455 \\
\hline Low & $€ 2.253 .442$ & $€ 2.293 .217$ & $2 \%$ & 7913 & 17877 \\
\hline Intermediate & $€ 4.218 .436$ & $€ 4.038 .096$ & $-4 \%$ & 11869 & 22538 \\
\hline High & $€ 32.731 .395$ & $€ 9.206 .204$ & $-72 \%$ & 29673 & 9040 \\
\hline PE management & $€ 16.510 .271$ & $€ 8.255 .135$ & $-50 \%$ & 9568 & 9568 \\
\hline Low & $€ 1.792 .778$ & $€ 1.314 .617$ & $-27 \%$ & 1039 & 1524 \\
\hline Intermediate & $€ 2.689 .167$ & $€ 3.386 .284$ & $26 \%$ & 1558 & 3925 \\
\hline High & $€ 12.028 .326$ & $€ 3.554 .234$ & $-70 \%$ & 6971 & 4119 \\
\hline $\begin{array}{c}\text { No PE } \\
\text { management }\end{array}$ & $€ 34.638 .582$ & $€ 23.758 .312$ & $-31 \%$ & 39887 & 39887 \\
\hline Low & $€ 2.096 .570$ & $€ 7.004 .825$ & $234 \%$ & 6874 & 14378 \\
\hline Intermediate & $€ 3.924 .772$ & $€ 10.423 .566$ & $166 \%$ & 10311 & 17696 \\
\hline High & $€ 28.617 .240$ & $€ 6.329 .921$ & $-78 \%$ & 22702 & 7812 \\
\hline Complications & $€ 23.108 .030$ & $€ 28.864 .826$ & $25 \%$ & 1704 & 2909 \\
\hline $\begin{array}{l}\text { Cost of } \\
\text { emergency } \\
\text { admissions }\end{array}$ & $€ 933.866$ & $€ 1.958 .976$ & $110 \%$ & 635 & 1612 \\
\hline $\begin{array}{c}\text { Neo-natal } \\
\text { intensive care }\end{array}$ & $€ 22.174 .164$ & $€ 26.905 .850$ & $21 \%$ & 1069 & 1297 \\
\hline Total & $€ 117.924 .952$ & $€ 84.764 .875$ & $-28 \%$ & 49455 & 49455 \\
\hline
\end{tabular}

The expected annual cost-savings for the INHS are equal to $€ 33.2$ million based on a cohort of 49 455 women with clinical signs of PE. Over 5 years, the cumulated savings are about $€ 159$ million 
( $3 \%$ discount rate). Net cost-saving per patient is equal to $€ 671$. Major healthcare costs avoided are related to hospitalization before the onset of $\mathrm{PE}$ (net cost-saving of $€ 476$ per patient) because the test helps assigning patients to the most appropriate management setting given the risk level detected (69.5\% reduction in unnecessary patient hospitalization in pre-PE management). As for complications, when the test is introduced, and compared to the alternative scenario, the increase in resources ( $€ 116$ per patient) is the result of the augmented number of patients managed in low and intermediate settings. Moreover, we assume that the test has no effect on frequency and LOS of EAs or admission to nICU. When introducing the test, patients who do not develop PE register fewer healthcare costs (net cost-saving of $€ 220$ per-patient) primarily because of a shift in clinical management setting from high intensity to intermediate/low intensity. In addition, the use of the sFlt-1/PIGF test during PE management allows fewer repeated hospitalizations for false-positive women (net cost-saving of $€ 167$ per-patient). The increased cost compared to standard practice is the cost of administering the sFlt-1/PlGF test and re-test that on average is equal to $€ 78.54$, considering $€ 50.12$ for each administration of the test and of which $81.36 \%$ of the patients are subject to re-test.

Sensitivity analysis

Sensitivity analysis was performed to test the robustness of model results (input variables were increased/decreased by $20 \%$ as standard range). The analysis suggested that 5 -year net savings per patient is sensitive to change in hospital admission costs for pregnant women with suspected PE (Figure 2). After the variation of the parameters, 5-year per-capita savings range between a minimum of $€ 497$ and a maximum $€ 773$.

Figure 2. Sensitivity analysis.

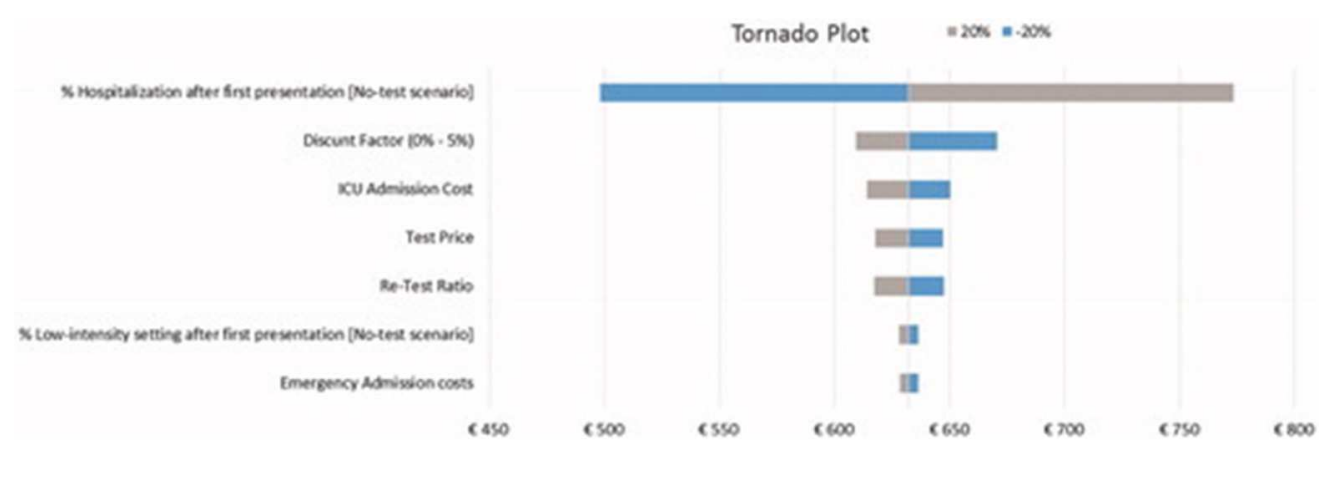

\section{Discussion}

There is a growing recognition of comprehensive economic assessment to inform regulators and payers when new health technologies are launched. Together with cost-effectiveness analysis (CEA), budget impact analysis (BIA) is the health economic technique used to manage and plan the 
introduction of new technologies/interventions on national, regional or local health budgets under resource constraints. Although CEA evaluates the costs and outcomes of alternative technologies in terms of health benefits to patients providing a measure of economic efficiency, BIA addresses the financial stream of consequences related to the uptake and diffusion of technologies giving a foundation for their affordability $[\underline{24}, \underline{25}]$. In particular, a BIA tries to foretell how a change in the mix of healthcare services, drugs and other therapies used to treat or predict a particular health condition will impact the trajectory of spending on that condition.

In this analysis, the budget impact of the novel PE biomarker Elecsys sFlt-1/PlGF immunoassay ratio was modeled as a supplementary diagnostic strategy to standard practice. The economic model was aimed at quantifying the net financial impact of the sFlt-1/PlGF test for the short-term prediction of PE for pregnant women with suspected PE in the perspective of the INHS. The results from the analysis showed how the new prenatal ratio test provides a more accurate prediction of PE development between gestational weeks $24+0$ until $36+6$ and consequently it is able to optimize the patient management and reduce unnecessary healthcare costs.

Overall, the introduction of the sFlt-1/PlGF ratio test helps addressing patients with low/intermediate risk of PE toward an outpatient setting while ensuring a more intense intervention to high risk PE patients to mitigate morbidity (hospitalization with corticosteroids for fetal lung maturation). Besides increasing costs, unnecessary hospitalization may also lead to decreased quality of life and might be associated with inappropriate and excessive intervention. The model results show how women in whom PE has been ruled out thanks to a PlGF-based test return to community care instead of being admitted to hospital for observation, with the main consequence of significant healthcare cost-savings. Based on the assumptions used to run the budget impact model, with the test and re-test, $24.3 \%$ of women were hospitalized before diagnosis of PE of which the $33.4 \%$ developed PE. Contrarily, without the test information, $60 \%$ of women were hospitalized before diagnosis of PE, of which only $26.4 \%$ went on to develop PE. The overall expectation of the positive value of the ratio test, in terms of a reduction of costs, is robust to plausible changes in the main parameters.

A similar study addressing the economics of diagnostic PE testing in the UK's context [18] is available. The results suggest similarities with the Italian context despite the more stringent clinical guidelines that are in place for the management of hypertensive pregnant women [15]. The BIA conducted in the UK report substantial cost-savings from a UK's healthcare payer-perspective, with savings of $£ 344$ per pregnant woman using the sFlt-1/PIGF test as supplementary diagnostic tool in comparison to standard practice [18]. 
National Institute for Health and Care Excellence (NICE) has recently published new evidencebased recommendations in which midwives and doctors in England and Wales were advised to use the Roche Elecsys ${ }^{\circledR}$ sFlt-1/PlGF immunoassay ratio test as one of two recommended tests to help rule-out PE together with standard clinical assessment and subsequent clinical follow-up, in women presenting with suspected PE between 20 and 34 weeks +6 days of gestation. The new guidance advocates the use of a test manufactured by Roche Diagnostics to confidently rule-out the presence of PE within 1 week [26].

The use in the UK has the potential to reduce hospitalization of suspected PE cases by $50 \%$, freeing up beds and reducing pressures on nurses. Annual UK savings are projected to be greater than $£ 16$ million [27].

The BIA we have presented has some limitations. First, the test scenario is based on management, resource use and outcome data collected from a large observational study (PROGNOSIS study), which includes pooled data from different countries but no data for Italy, thus predictive value may vary in actual clinical practice. Second, some resource utilization inputs, such as frequency of outpatient visits and patient allocation to the different levels of management, are an average value obtained from interviews to National Experts. Moreover, patient pathways may also vary across the country since the INHS is decentralized at regional levels and organization and provision of health services are not homogeneous [25]. Due to the fragmented national scenario, the increase in litigation might have a role in unnecessary hospitalization in certain regions. Third, the model assumes the uptake of the Elecsys sFlt-1/PIGF test for the entire target population of pregnant women with suspected PE starting from the first year of test's availability, but adoption rate may change across regions/hospitals especially if stringent national clinical guidelines are not in place. However, the limitations of the model are not expected to significantly impact the findings of the analysis. Results remain robust to changes in main parameters in the economic model as shown in the sensitivity analysis. In addition, model estimations are conservative because they focus on maternal health and do not include follow-up management and costs for women and infants after delivery and long-term complications.

\section{Conclusion}

The introduction of prenatal sFlt-1/PIGF ratio test in addition to standard practice may be useful to prevent over-diagnosis and over-treatment of women with suspected PE avoiding unnecessary hospitalizations across all risk levels. At the same time, it allows better identification of women at higher PE risk who can receive an appropriate management accumulating cost-savings to the INHS. 


\section{Declaration of interest}

Davide Paolini and Matteo Dionisi reported being employees of Roche Diagnostics S.p.A. The research has been supported by an unconditional grant from Roche Diagnostics S.p.A.

\section{Notes}

1 Suspicion of clinical diagnosis of PE implicates new onset of elevated blood pressure; aggravation of preexisting hypertension; new onset of protein in urine; one or more of the following reasons, such as epigastric pain; severe edema; headache; visual disturbances; weight gain; low platelets; elevated liver transaminases; suspected intrauterine growth restriction; abnormal uterine perfusion detected by Doppler sonography with mean Pulsatility Index $(\mathrm{PI})<95$ th percentile in the second trimester and/or bilateral uterine artery notching.

2 Population growth was calculated as the average compound annual growth rate of female population (2012-2105).

3 PE signs are identified as epigastric pain; severe edema; headache; oliguria; visual disturbances; confirmed hypertension; confirmed proteinuria; proteinuria dipstick result of +1 ; one or more criteria met for HELLP syndrome: increased AST (<70 IU/L); reduced thrombocyte counts $(<100,000 / \mu \mathrm{L})$; increased LDH levels (>600 IU/L); intrauterine growth restriction; abnormal uterine perfusion detected by Doppler Sonography with mean PI $>95$ th percentile.

4 Exchange rate Pound to Euro was applied (Bank of Italy 26 August 2015).

\section{References}

1. Khan KS, Wojdyla D, Say L, et al. WHO analysis of causes of maternal death: a systematic review. Lancet 2006;367:1066-74

2. Duley L. The global impact of pre-eclampsia and eclampsia. Semin Perinatol 2009;33:130-7

3. Society of Obstetricians and Gynecologist of Canada (SOGC) Guideline. J Obstet Gynaecol Can 2014;36:416-38

4. Royal College of Obstetricians and Gynecologists (RCOG) Guideline. The management of severe pre-eclampsia/eclampsia. Guideline No. 10(A). 2010. [Internet]. Available from: http://ncagip.ru/for-experts/snk/RCOG.pdf [Google Scholar]

5. Italian Association of Preeclampsia (AIPE) Guideline 2013. [Internet]. Available from: http://www.preeclampsia.it/images/linee_guida/Linee_Guida_AIPE_2013.pdf

6. Levine RJ, Maynard SE, Qian C, et al. Circulating angiogenic factors and the risk of preeclampsia. N Engl J Med 2004;350:672-83 
7. Sibiude J, Guibourdenche J, Dionne M-D, et al. Placental growth factor for the prediction of adverse outcomes in patients with suspected preeclampsia or intrauterine growth restriction. PLoS One 2012;7:e50208

8. Larroca SG-T, Tayyar A, Poon LC, et al. Competing risks model in screening for preeclampsia by biophysical and biochemical markers at 30-33 weeks' gestation. Fetal Diagn Ther 2014;36:9-17

9. Cetin I, Mazzocco MI, Giardini V, et al. PlGF in a clinical setting of pregnancies at risk of preeclampsia and/or intrauterine growth restriction. J Matern Fetal Neonatal Med 2017;30:144-9

10. Lapaire O, Shennan A, Stepan H. The preeclampsia biomarkers soluble fms-like tyrosine kinase-1 and placental growth factor: current knowledge, clinical implications and future application. Eur J Obstet Gynecol 2010;151:122-9

11. Zeisler H, Llurba E, Chantraine F, et al. Predictive value of the sFlt-1:PlGF ratio in women with suspected preeclampsia. N Engl J Med 2016;374:13-22

12. Hund M, Allegranza D, Schoedl M, et al. Multicenter prospective clinical study to evaluate the prediction of short-term outcome in pregnant women with suspected preeclampsia (PROGNOSIS): study protocol. BMC Pregn Childbirth 2014;14:324

13. Fattore G. Proposta di linee guida per la valutazione economica degli interventi sanitari in Italia. Pharmacoeconomics-Ital-Res-Articles 2009;11:83

14. National Institute of Statistics (ISTAT) [Internet]. Available from: http://dati.istat.it/

15. National Institute for Health Care Excellence (NICE). Hypertension in pregnancy: the management of hypertensive disorders during pregnancy. Royal College of Obstetricians and Gynecologists. First published August 2010; revised reprint January 2011. [Internet]. Available from: https://www.nice.org.uk/guidance/cg107/evidence/full-guideline-134794333

16. Borghi C, Ferri C, Sechi L. Italian Society of Hypertension. Clinical management of hypertension in pregnancy. Practical recommendations from the Italian Society of Hypertension (SIIA). High Blood Press Cardiovasc Prev 2013;20:123-7

17. Mammaro A, Carrara S, Cavaliere A, et al. Hypertensive disorders in pregnancy. J Prenat Med 2009;3:1-5

18. Vatish M, Strunz-McKendry T, Hund M, et al. The sFlt-1/PlGF ratio test in pre-eclampsia: an economic assessment for the UK. Ultrasound Obstet Gynecol 2016;48:765-71

19. Regione Liguria DGR $n^{\circ} 1308$ del 24/10/2013, Approvazione del catalogo unico regionale delle prestazioni sanitarie ambulatoriali erogabili sul territorio ligure, National code number 90.53.5: sFlt-1 (Regional code number: C01831800) PlGF (Regional code number: C01850700)

20. Remunerazione delle prestazioni di assistenza ospedaliera per acuti, assistenza ospedaliera di riabilitazione e di lungodegenza post acuzie e di assistenza specialistica ambulatoriale. Decreto 
Ministeriale del 18 ottobre 2012. Supplemento ordinario alla "Gazzetta Ufficiale" n. 23 del 28 gennaio 2013

21. Xiao R, Sorensen TK, Williams WA, Luthy DA. Influence of pre-eclampsia on fetal growth. J Matern-Fetal Neonat Med 2003;13:157-62

22. Payment by results in the NHS: Tariff Information Spreadsheet [Internet]. Available from: https://www.gov.uk/government/publications/payment-by-results-pbr-operational-guidanceand-tariffs [last accessed Feb 2014]. Exchange rate Pound to Euro, Bank of Italy, 26-08-2015

23. Tan SS, Bakker J, Hoogendoorn ME, et al. Direct cost analysis of intensive care unit stay in four European countries: applying a standardized costing methodology. Value Health 2012;15:81-6

24. Mauskopf JA, Sullivan SD, Annemans L, et al. Principles of good practice for budget impact analysis: report of the ISPOR Task Force on good research practices-budget impact analysis. Value Health 2007;10:336-47

25. Ferré F, de Belvis AG, Valerio L, et al. Italy: Health system review. Health Syst Transit 2014;16:1168

26. National Institute for Health and Care Excellence. PIGF based testing to help diagnose suspected pre-eclampsia. NICE Guideline [Internet]; 2016. Available from: https://www.nice.org.uk/guidance/dg23/resources

27. Data on File: DOF_sFlt/PIFG_05_16 (Adapted from National Institute for Health and Care Excellence) PlGF based testing to help diagnose suspected pre-eclampsia. NICE resource impact template [Internet]; 2016. Available from: https://www.nice.org.uk/guidance/dg23/resources 